\title{
Transcription Elongation
}

National Cancer Institute

\section{Source}

National Cancer Institute. Transcription Elongation. NCI Thesaurus. Code C28586.

The RNA polymerase moves along the DNA template, adding nucleotides to the growing RNA chain in accordance with the sequence of the template or coding strand. 